\title{
HIGH EFFICIENCY P-I-N SOLAR CELLS WITH <I> LAYER DEPOSITED BY HOT-WIRE TECHNIQUE
}

\author{
Y. Ziegler, S. Dubail, C. Hof, U. Kroll, and A. Shah. \\ Institute of Microtechnology, University of Neuchâtel, A.-L. Breguet 2, CH-2000 Neuchâtel, Switzerland
}

\begin{abstract}
More stable amorphous silicon (a-Si:H) material obtained with the Hot-Wire technique requires high substrate temperatures $\left(\mathrm{T}_{\text {sub }}>300^{\circ} \mathrm{C}\right)$ and low gas pressures $\left(p_{\text {dep }} \approx 10^{-2}\right.$ mbar $)$. The second condition implies that heat transfer between the heater and the substrate is mainly dominated by thermal radiation; therefore, $T_{\text {sub }}$ is strongly affected by the emissivity $\varepsilon_{\text {surf }}$ of the growth surface. Here, it will be shown experimentally that $\varepsilon_{\text {surf }}$ depends on the thickness of the growing a-Si: $\mathrm{H}$ layer; a significant variation in $\varepsilon_{\text {surf }}$ during the deposition of the first $3000 \AA$ has been observed. With the help of a new heating concept, we integrated an intrinsic layer deposited at constant $\mathrm{T}_{\text {sub }}=270^{\circ} \mathrm{C}$ into a $\mathrm{p}$ i-n solar cells with initial efficiency of $8.7 \%$.
\end{abstract}

\section{INTRODUCTION}

Several groups have successfully demonstrated in the past that the hot-wire technique is an encouraging alternative to produce more stable a-Si:H [1-4]. By analysing the different publications, we observe that the deposition of this material requires generally two typical deposition parameters, i.e. high substrate temperature (up to $400{ }^{\circ} \mathrm{C}$ ) and low gas pressure ( $p_{\text {dep }} \approx 10^{-2}$ mbar). However, at elevated temperature the integration of such material into $\mathrm{p}$-i-n solar cell remains difficult, mainly due to the intrinsic layer contamination caused by the diffusion of impurities from the substrate (sodium) and from the doped layer (boron).

To reduce the contamination, Mahan et al. [5] realised $n$-i-p solar cell on stainless steel, with $7 \%$ initial efficiency $\left(T d e p \approx 370^{\circ} \mathrm{C}\right.$ ), but it is not clear if the performance of this solar cell is limited by the properties of the intrinsic layer or by thermal diffusion effect (phosphorus, hydrogen).

Simultaneously, Bauer et al. [6] proposed to evaluate the possibility to realise high efficiency $p-i-n$ solar cells with an intrinsic layer deposited by hot-wire at moderate temperature $\left(220-260^{\circ} \mathrm{C}\right)$. By controlling the $p$ $i$ interface with in-situ ellipsometry measurement, they obtained about $10 \%$ initial efficiency; this clearly demonstrates the compatibility between the hot-wire technique and the $p-i-n$ fabrication technology.
The topic of the present study concerns the problem of controlling the substrate temperature during the deposition of the intrinsic layer by hot-wire. The difficulty results mainly from two factors: the first one is the thermal radiation emitted by the filament. The second is related to the fact that the deposition is realised at low gas pressure $\left(p_{d e p} \approx 10^{-2} \mathrm{mbar}\right.$ ); in this case, the substrate heater (i.e. substrate holder acting as hot plate) operates in the thermal radiation regime and not in the thermal conduction one (see Fig. 1). Thus, the deposition temperature depends mainly on the surface emissivity $\varepsilon_{\text {surf }}$ of the substrate.

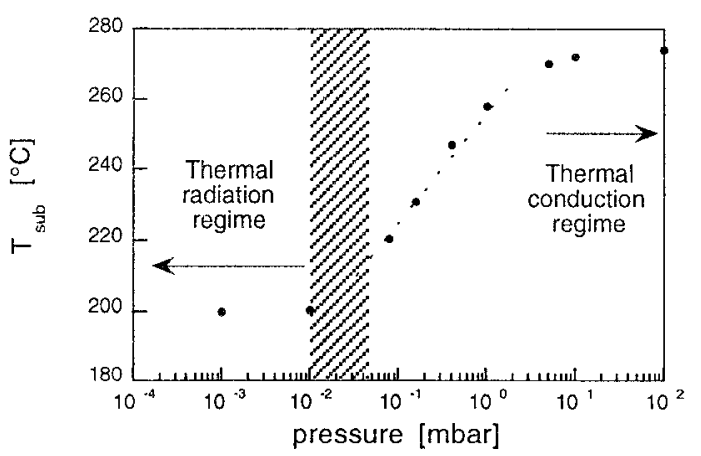

Fig. 1. Influence of the gas pressure on the substrate temperature $T_{\text {sub }}$ heated by a hot plate at constant temperature of $275^{\circ} \mathrm{C}$. The dashed area represents the typical pressure range used to produced more stable aSi:H with hot-wire technique.

This work will show how the surface emissivity $\varepsilon_{\text {surf }}$ depends on the thickness of the a-Si:H deposited on the substrate. Thus, the substrate temperature $T_{\text {sub }}$ tends to vary during the deposition of the solar cell intrinsic layer.

\section{EXPERIMENTAL}

\section{New heating system}

Due to substantial thermal inertia, the conventional heater is not effective in compensating a fast change of the substrate temperature. To improve the response time 
of the substrate, we have integrated a low mass heater onto the substrate. The heating element is realised by a thin metallic film (chromium) deposited on the whole surface of one side of the substrate (see fig. 2). As already described in detail [7], the substrate temperature $T_{\text {sub }}$ is controlled by the electrical power $\mathrm{Pel}^{\mathrm{el}}$ applied to the thin metallic film.

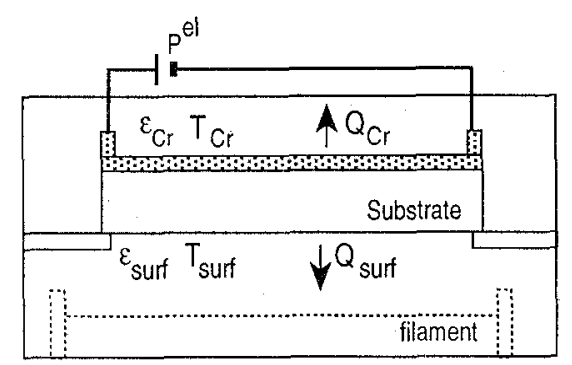

Fig. 2. Cross-section of the glass substrate and the metallic film acting as heating element. $Q_{C r}$ represents the radiative losses emitted from the metallic surface, and $Q_{\text {surf }}$ those emitted from the surface of the substrate.

\section{Films and cells preparation}

Device-quality a-Si: $H$ films have been prepared in a UHV apparatus using the following deposition parameters: gas pressure of $10^{-2} \mathrm{mbar}$, hydrogen and silane flow rate of $10 \mathrm{sccm}$; the gas is decomposed by a tungsten filament with a length of $150 \mathrm{~mm}$ and a diameter of $0.3 \mathrm{~mm}$ diameter, heated at $1850^{\circ} \mathrm{C}$ and placed at a distance of $5 \mathrm{~cm}$ from the substrate.

The solar cells, with an intrinsic layer of $4000 \AA$, were deposited on $\mathrm{SnO}_{2}$-coated glass substrate (U2 from Asahi). The doped layers $\langle p\rangle$ and $\langle n>$ were obtained within a separate PE-CVD reactor; this implied a transfer from one system to the other one by exposing each interface of the solar cell to the air. Finally, a sputtered ITO/Ag has been used for the back contact.

\section{RESULTS AND DISCUSSION}

\section{Surface emissivity $\varepsilon_{\text {surf }}$}

At low deposition pressure the substrate temperature $T_{\text {sub }}$ depends strongly on the surface emissivity $\varepsilon_{\text {surf }}$ in both heating systems. Therefore, it is necessary to determine how $\varepsilon_{\text {surf }}$ is influenced by the deposited a-Si:H film, this being important where the initial value of $\varepsilon_{\text {surf }}$ differs from the value for $\mathrm{a}-\mathrm{Si}: \mathrm{H}\left(\varepsilon_{\mathrm{a}-\mathrm{Si}: \mathrm{H}}\right.$ $\approx 0.6$ ). We have analysed two typical cases (see Fig. 3.): the deposition of a-Si:H on glass $\left(\varepsilon_{\text {glass }} \approx 0.9\right.$ ) and on TCO $\left(\varepsilon_{\mathrm{TCO}} \approx 0.3\right)$. The determination of $\varepsilon_{\text {surf is evaluated }}$ from $T_{\text {sub }}$, measured with a Pt100 pasted on the surface of the substrate, and using the relation derived from the Stefan-Bolzmann law [8]:

$$
\varepsilon_{\text {surf }}=\frac{P^{\mathrm{el}}+A_{\mathrm{s}}^{\text {reac }}}{\sigma \cdot T_{\mathrm{s}}^{4}}-\varepsilon_{\mathrm{cr}}
$$

where $\mathrm{P}^{\mathrm{el}}$ is the electrical power density applied to heat the substrate, $A^{\text {reac }}$ the thermal power contribution of the internal wall of the reactor determined experimentally and $\sigma$ the Stefan-Bolttzmann constant.

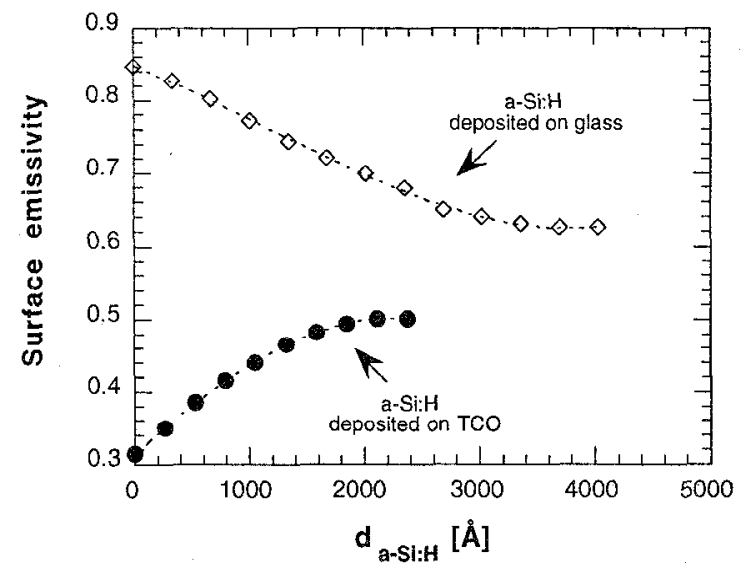

Fig. 3. Measured effect of the a-Si:H thickness on the surface emissivity $\varepsilon_{\text {surf }}$ for the cases of a-Si:H deposited on glass substrate (Corning 7059) and on TCO substrate $\left(\mathrm{SnO}_{2}\right.$ textured). The dashed lines are the interpolated functions used for the simulations presented in the next section.

In both cases, we observed, first, that the surface emissivity depends on the thickness of the deposited a-Si:H ( noted $d_{a-S i: H}$ in fig. 3 ) and, secondly, that $\varepsilon_{\text {surf }}$ saturated above a certain thickness, depending on the type of the substrate. Consequently, at a given electrical power $\mathrm{P}^{\mathrm{el}}$ in our "new" heating system, or, at a fixed temperature of the hot plate (conventional heating system), the temperature of the glass substrate is not equivalent to that of the TCO; this will be discussed in the next section.

\section{Substrate temperature: measure and simulation}

Both simulations and experimental results of the substrate temperature variation during the deposition of a-Si:H film using the Hot-Wire technique and at low gas pressure will be presented. The effect of the filament radiation on the substrate (but not on the growing layer) as well as the influence of the growing a-Si:H film on the surface emissivity have both been considered in our simulations.

Two situations are analysed in the present paper, i.e. the deposition of a-Si:H on glass and on TCO (see Fig. 4). In the first case, the substrate temperature increases monotonously, this due to the decrease of $\varepsilon_{\text {sur }}$ with the thickness of the deposited a-Si:H film. However, 
the saturation above $=400 \mathrm{C} \AA$ of deposited a- $\mathrm{Si}: \mathrm{H}$ is not reached, as predicted theoretically from the simulation. This effect is attributed to the absorbed part of the emitted filament radiation by the a-Si:H, which increases with the thickness of the deposited a-Si:H film.

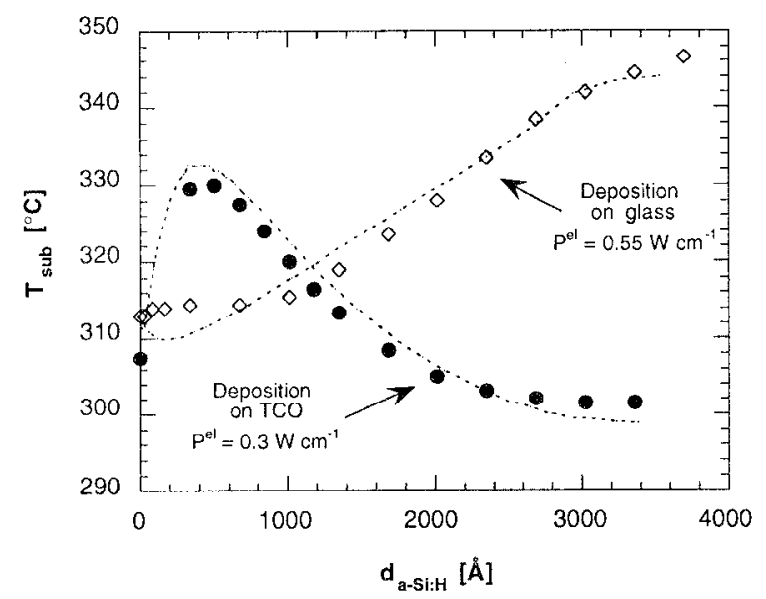

Fig. 4. New heating system: measurements (symbols) and simulations (dashed lines) of the substrate temperature $T_{\text {sub }}$ during the deposition of a-Si:H on glass substrate (open symbols) and on TCO (plain circles) when the electrical power was set at constant values (in this case, $P^{\mathrm{el}}$ was set to obtain the same temperature on glass and on TCO prior to the deposition).

In the second case (i.e. deposition on TCO), we observe initially a fast increase of $T_{\text {dep }}$ due to the absorption of the filament radiation by the TCO and finally a slow decrease of $T_{\text {dep }}$ caused by the increase of $\varepsilon_{\text {surt }}$. In both situation, the simulations and measurements agree well, although we neglect the contribution of the absorption of the emitted filament radiation by the a-Si:H. The changes of the substrate temperatures are also significant and very different in both situations. That has the consequence that it is difficult to simultaneously deposit identical layers on glass and on TCO as is done often to correlate film properties and solar cell performances. Similar behaviour are also observed in the case of deposition at low pressure with a conventional heating system.

\section{Solar cells}

Finally, the effect of the temperature variation during the deposition of the intrinsic layer incorporated into a $p$-i-n structure by hot-wire technique has been analysed in three situations (see Fig. 5):

- Cell \#1: the electrical power $P^{e t}$ applied to heat the substrate was set to the value (i.e. $0.4 \mathrm{Wcm}^{-1}$ ) used to obtain $270^{\circ} \mathrm{C}$ on glass substrate; that means we do not consider the difference of the emissivity between the glass and the TCO. Hence, the temperature before the deposition is higher than the expected value of $270^{\circ} \mathrm{C}$, because the emissivity of TCO is smaller than that of the glass. Secondly, a strong increase of $T_{\text {dep }}$ up to $350^{\circ} \mathrm{C}$ in the initial phase of the deposition occurs, due to the absorption of the filament radiation by the TCO ( the $\mathrm{SnO}_{2}$ is not fully transparent in the infra-red region).

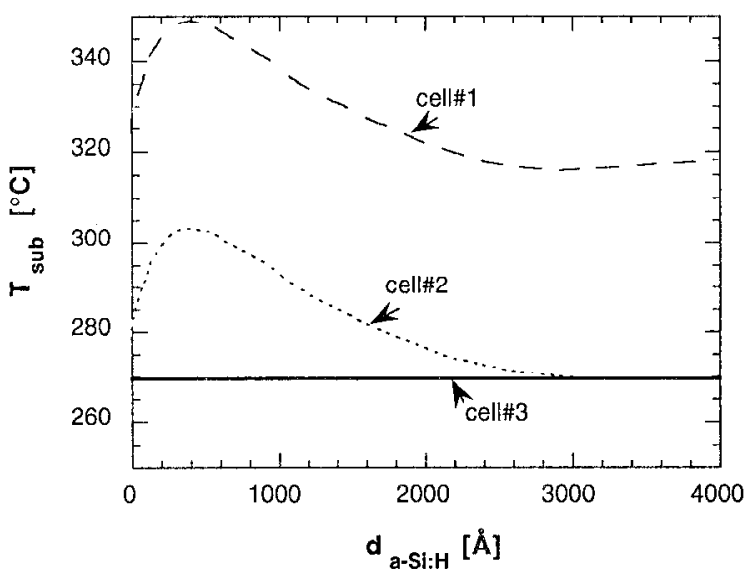

Fig. 5. Simulation of the substrate temperature during the growth of the intrinsic layer for the three cases described in the text.

- Cell \#2: the value of the TCO emissivity was taken into account by adjusting the electrical power to obtain $270^{\circ} \mathrm{C}$ prior to the deposition $\left(\mathrm{P}^{\mathrm{el}}=0.2 \mathrm{Wcm}^{-1}\right)$. Now, the substrate temperature rises up to $300^{\circ} \mathrm{C}$ during the initial phase of the deposition and decreases monotonously to $270^{\circ} \mathrm{C}$ after.

- Cell \#3: By adapting the electrical power, the substrate temperature was kept at a constant value of $270^{\circ} \mathrm{C}$; note that the low thermal inertia of the heating system was able to compensate the fast increase of $T_{\text {dep }}$ that occurs during the initial phase of the deposition (effect of the radiation of the filament).

Table 1 summarizes the performances of the three solar cells described above. First, it has been observed that the $V_{o c}$ and the fill factor of the cell $\# 1$ are strongly reduced by the high substrate temperature during the deposition of the intrinsic layer.

\begin{tabular}{|l|c|c|c|c|}
\hline & $\begin{array}{c}V_{\text {oc }} \\
{[\mathrm{V}]}\end{array}$ & $\begin{array}{c}\mathrm{I}_{\mathrm{sc}} \\
{\left[\mathrm{mAcm}^{-1}\right]}\end{array}$ & $\mathrm{FF}$ & $\begin{array}{c}\eta \\
\%]\end{array}$ \\
\hline Cell \#1 & 0.680 & 13.26 & 0.540 & 4.87 \\
\hline Cell \#2 & 0.824 & 15.24 & 0.646 & 8.11 \\
\hline Cell \#3 & 0.838 & 14.85 & 0.704 & 8.76 \\
\hline
\end{tabular}

Table 1. Summary of solar cells performances obtained under different heating conditions applied during the growth of the intrinsic layer. 
In cell \#2, the moderate fill factor can probably also be attributed to the increase of $T_{\text {dep }}$ during the initial phase of the deposition; this hypothesis is supported by the results obtained for the cell \#3, where the substrate temperature was kept constant.

Finally, it is important to evaluate the potential of improving the solar cell efficiency containing an intrinsic layer deposited by hot-wire. In the past, there has been found a strong correlation between the solar cell performance and the quality of the intrinsic layer evaluated with $\mu^{0} \tau^{0}$ [9]. We observe that the cell \#3 is close to the correlation obtained for full PE-CVD solar cells (see Fig. 6). That means that the performance of the cell \#3 is mainly limited by the quality of the intrinsic aSi:H layer.

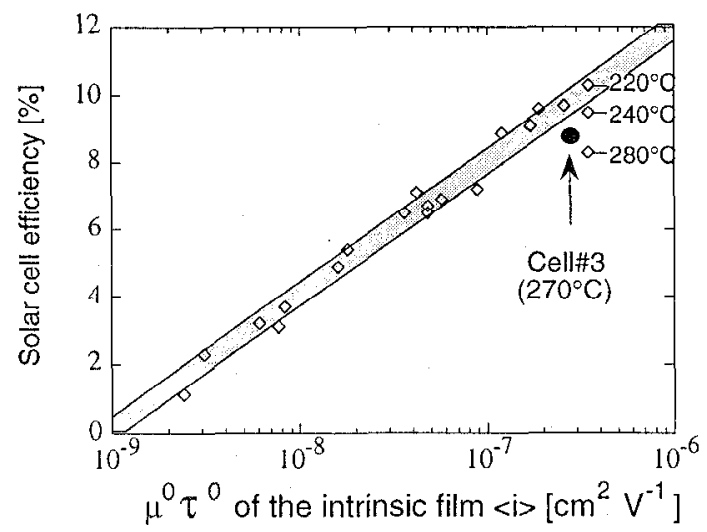

Fig. 6. Correlation between the intrinsic layer quality (characterised with the so-called $\mu^{0} \tau^{0}$ parameter) and the solar cell performance. Open symbols represent a study with an intrinsic layer deposited by PE-CVD and the circle the cell \#3; the doped layers $<p>$ and $<n>$ were identical for all solar cells. Note that an increase of the deposition temperature affects the performance of the solar cell, although the quality of the intrinsic layers deposited between $220^{\circ} \mathrm{C}$ and $280^{\circ} \mathrm{C}$ by PE-CVD romains constant.

\section{CONCLUSIONS}

In the present study, the problem of keeping the substrate temperature constant during the deposition of intrinsic a-Si:H layers with the hot-wire technique at low pressure $\left(<10^{-1}\right.$ mbar) was considered. The radiation of the filament and the change of the surface emissivity during the growth affected strongly $T_{\text {dep. }}$. We have also demonstrated that a better control of the substrate temperature during the deposition of the intrinsic layer can improve significantly the solar cell performances, especially the fill factor.

The substrate temperature in the case of solar cell with intrinsic layer deposited by hot wire is a crucial point, because the more stable materials obtained with this technique require a relatively high $\mathrm{T}_{\text {sub }}$. However, it has been shown recently that more stable a-Si:H can be obtained at moderated $\mathrm{T}_{\text {sub }}$, i.e. $320^{\circ} \mathrm{C}$ [4]; thus both a good control of the deposition temperature and a development of an anti-diffusion barrier (integrated into the $\langle\mathrm{p}\rangle$ layer) will certainly allow to realise more stable solar cells.

\section{ACKNOWLEDGEMENTS}

This work was supported by the Swiss Federal Department of Energy BEW / OFEN, project No. 19431

\section{REFERENCES}

1] A. H. Mahan, M. Vanecek, "A reduction in the StaeblerWronski effect observed in low $\mathrm{H}$ content a-Si:H films deposited by the hot wire technique", AIP Conf. Proc. 234, 1991 , pp. $195-202$

[2] R. Zedlitz, F. Kessler, and M. Heintze, "Deposition of a-Si:H with Hot-Wire technique", J. Non-Cryst. Solids 164-166, 1993 , pp. $83-86$

[3] P. Papapdopulos, A. Scholz, S. Bauer, B. Schröder, $\mathrm{H}$. Oechsner, "Deposition of device quality a-Si:H films with the hot wire technique". J. Non-Cryst. Solids 164166, 1993, pp. $87-90$

[4] C. Hof, Y. Ziegler, R. Platz, N. Wyrsch, and A. Shah, "Stability of a-Si:H prepared by Hot-Wire and Glow Discharge using $\mathrm{H}_{2}$ dilution evaluated by pulsed laser degradation", to be published in proceedings of the $17^{\text {th }}$ International Conference on Amorphous Semiconductors, Budapest, 1997

[5] B. P. Nelson, E. Iwaniczko, R. Schropp, A. H. Mahan, E. Molenbroek, S Salamon, and R. S. Crandall, "Amorphous Silicon Solar Cells Incorporating Hot Wire Deposited Intrinsic Layers", $12^{\text {th }}$ European Photovoltaic Solar Energy Conference, 1994, pp. 679 - 682

[6] S. Bauer, W. Herbst, B. Schroeder, H. Oechsner, W. Frammelsberger, and H. Schade, "An Approach towards High Efficiency Hot-Wire CVD based a-Si:H PIN Solar Cells", presented at the $14^{\text {th }}$ EC PVSEC, Barcelona, 1997

[7] Y. Ziegler, M. Goerlitzer, and A. Shah, "New results on Hot-Wire a-Si:H: Improvement of Film Homogeneity and More Reliable Criteria for Electronic Quality", $13^{\text {th }}$ European Photovoltaic Solar Energy Conference, 1995, pp. 206 - 209

[8] Y. Ziegler, Ph. D. Thesis, University of Neuchâtel, 1997

[9] N. Beck, N. Wyrsch, C. Hot, and A. Shah, J. Appl. Phys. 12, 1996, p. 9361 